\title{
Experimental Study on Performance Characteristics of Electrodynamic Rotors in Maglev Transportation System
}

\author{
Syed Hassaan Abdullah, Mirza Shuja Mohiuddin, Mohammed Hasnain, Mohammed Irfan Siddiq, \\ Mohammed Abdul Aziz
}

\begin{abstract}
When an array of rare-earth magnets, embedded in a The design was later improved by Bird and Lipo, by rotor, is subjected to mechanical rotation over a conductive, introducing electrodynamic wheels with the permanent paramagnetic guide-plate, where the axis of the rotor is magnets placed in Halbach array configuration. However, these perpendicular to the guideway, it induces eddy currents that proposed models cannot achieve a smooth planar motion by create a counter magnetic field resulting in levitation and drag creating levitation and propulsion concurrently, moreover, the forces. Since the net drag force in the guide-plate is zero, hence, system will always be restricted by the guide track. To rotors only generate levitation forces that stabilize the system system will always be restricted by the guide track. To
without creating any directional motion. This paper is an attempt overcome this, Park and Baek suggested a maglev planar without creating any directional motion. This paper is an attempt oransportation vehicle system by changing the orientation of the of rotors, number of magnetic poles and material $\&$ dimensions of rotor in order to create only levitation. Propulsion in MPTV is guide-plate) on a "quadcopter" working on electromagnetic achieved by employing four single-sided transverse flux linear levitation achieved by spinning electrodynamic rotors. Additional induction motors (STFLIM), each controlling its respective investigation on the effect of an increase in temperature of the directional motion. This proposed model was studied in detail guide plate on the lift of the quadcopter was also recorded.

Index Terms - Maglev Transportation, Electrodynamic rotor, Halbach Array, Magnetic Levitation, Hyperloop trains, Lenz Law. by designing, analyzing and fabricating a maglev planar transportation system, however, only the levitation characteristics were studied due to financial and laboratory constraints.
\end{abstract}

\section{INTRODUCTION}

Magnetic levitation technology is a non-contact wear-free system, which offers low maintenance, negligible slipping and minimal noise and vibrations over conventional mechanical systems. It is widely employed in high-speed maglev trains, Hyperloop pods, high precision devices, and frictionless bearings.

Electrodynamic rotors or wheels were traditionally used in prototypes of maglev trains, but, due to its low efficiency and small working range, they were not feasible at the time. However, due to radical advancements in the field of highpower permanent magnets, these rotors are the most plausible solution to the traditional electrodynamic suspension (EDS) model, by simultaneously creating levitation and propulsion forces. Fujiiet al. performed various experiments in order to strengthen the proposed design of using a magnetic wheel to produce levitation and propulsion in unison.

Syed Hassaan Abdullah, Undergraduate B.E. Student, Mechanical Engg. Department, Muffakham Jah College of Engg. \& Tech., India

Mirza ShujaMohiuddin, Undergraduate B.E. Student, Mechanical Engg. Department, Muffakham Jah College of Engg. \& Tech., India

Mohammed Hasnain, Undergraduate B.E. Student, Mechanical Engg. Department, Muffakham Jah College of Engg. \& Tech., India

Mohammed Irfan Siddiq, Undergraduate B.E. Student, Mechanical Engg. Department, Muffakham Jah College of Engg. \& Tech., India

Mohammed Abdul Aziz, Undergraduate B.E. Student, Mechanical Engg. Department, Muffakham Jah College of Engg. \& Tech., India

In this paper, the effects of rotational speed of electrodynamic rotors, number of magnetic poles and material \& dimensions of the platform, on the performance of the quadcopter have been characterized. Since, characteristics of metals changes with an increase in temperature, an additional investigation on the effect of the increase in temperature of the guide plate on the suspension of the quadcopter were also recorded.

\section{DESIGN AND DEVELOPMENT OF THE MAGLEV SYSTEM}

\section{A. Design Methodology :}

An air-propelled quadcopter was selected as a model for the maglev system's design, because of its simplicity. Based on the diameter of the electrodynamic rotor disc and circular pitch diameter of the rotors, the arm length of the quadcopter was selected and was designed in Autodesk Fusion 360.

Even though, 2-pole and 8-pole magnetic arrangement is being used the diameter of the electrodynamic rotor is fixed constant which has been optimally selected from the research of Bird and Lipo[2]. The rotors were 3D printed with $0.1 \mathrm{~mm}$ finish. 


\section{B. CAD Modelling :}

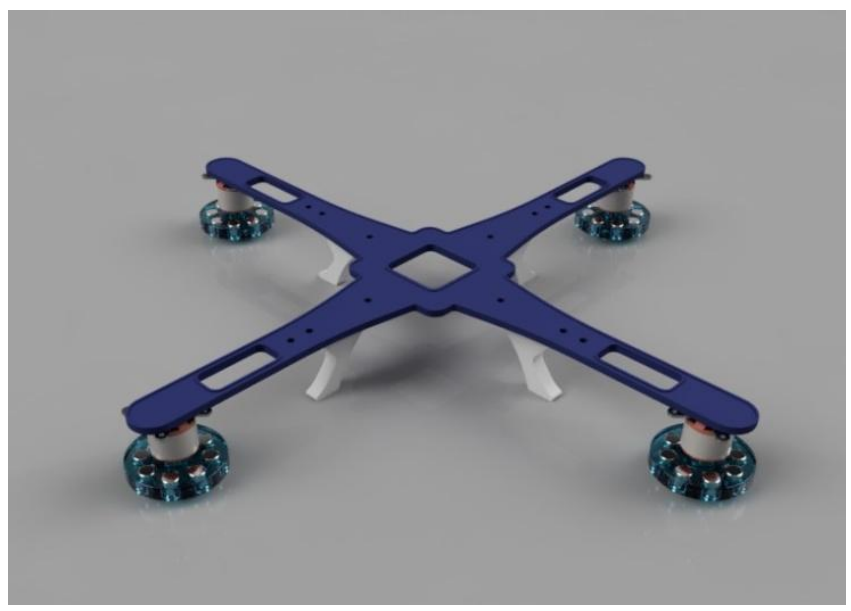

Fig. 1 : Rendering of the Maglev Quadcopter designed using Autodesk Fusion 360

\section{Levitation Mechanism :}

Levitation in the quadcopter is achieved by using the principle of Lenz's Law, which states that "the direction of the current induced in a conductor by a changing magnetic field is such that the magnetic field created by the induced current opposes the initial changing magnetic field".

The rotor employing the strong magnetic array is rotated at high speeds over a paramagnetic guide plate. This rotation induces eddy currents in the plate, which creates magnetic poles similar to the rotor, almost mirroring it. This results in magnetic repulsion. If the rotational speed is increased further the magnetic flux induced increases, hence, producing an upward lift. To improve the induced magnetic flux, the array was arranged in two different orientation, 2pole and 8-pole. Although the best orientation is that of a halbach array, it couldn't achieved because of limitations of the shape of the magnets.

2-pole magnetic array is the one in which half of the array is present in one orientation and vice versa. In 8-pole magnetic array, each magnet has one orientation while it's adjacent one has the opposite. The orientation of magnets have a great effect on the magnetic flux induced, henceforth generating greater lift.

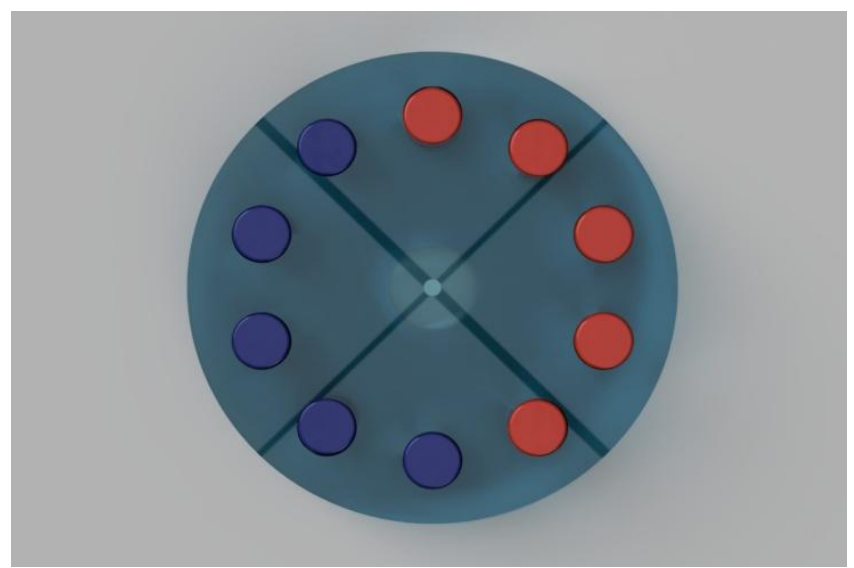

(a)

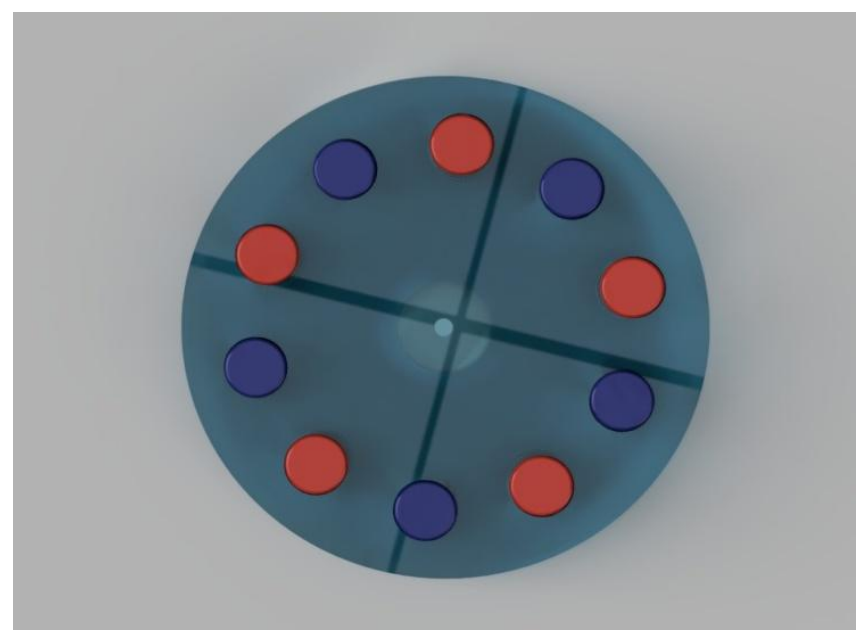

(b)

Fig .2 : Orientation of the array of magnets : (a) 2-pole (b) 8-pole

In the figures above, the red color on the magnet is representing the North pole while the blue one is representing the south pole of the magnet.

\section{EXPERIMENTAL SETUP}

The experimental setup comprised of the maglev quadcopter, guideplate, potentiometer, LiPo batteries, vernier height gauge, laser tachometer and infrared thermometer. Brushless DC motors were used to spin the electrodynamic rotors, as they have the capability to reach very high speeds of around 20,000 rpm. The potentiometer was used to vary the speed of the rotors from $10,000 \mathrm{rpm}$ to a maximum of $17,500 \mathrm{rpm}$ with increments of $500 \mathrm{rpm}$. Vernier height gauge with a least count of $0.02 \mathrm{~mm}$ was incorporated to measure the lift height of the maglev system, four verniers were placed on four edges of the quadcopter and the average height was taken. The experimental setup is shown in figure 3.

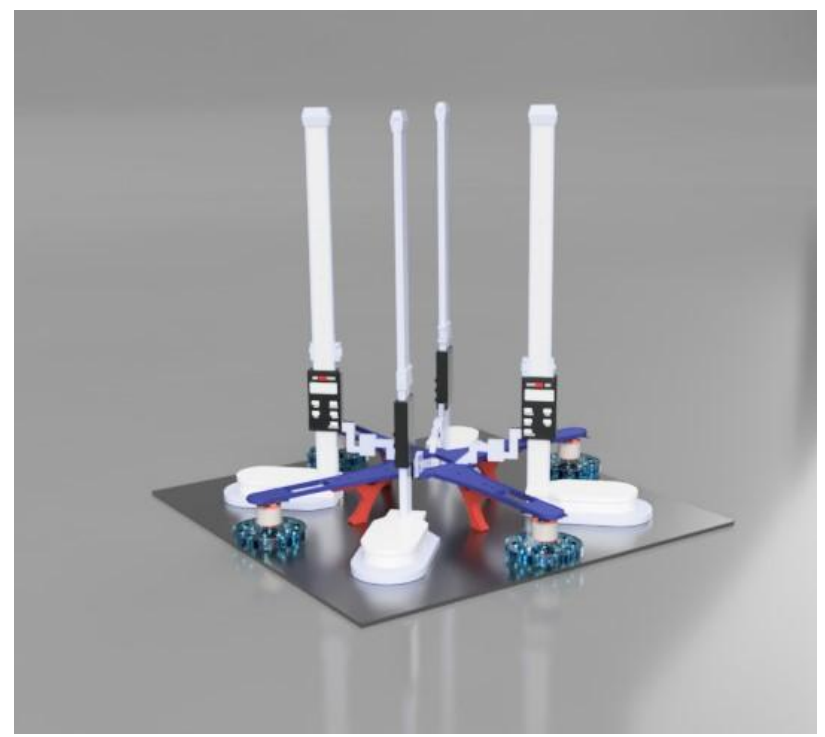

Fig. 3 : Experimental setup of the investigation

A much more sophisticated height gauge could have been employed, however, due to financial constraints the options were limited to standard gauge with $0.02 \mathrm{~mm}$ least count. 
Before initiating the experiment laser tachometers were used to set the rotational speeds of all the rotors same, as to avoid any instability and drag. Increase in temperature of the guide plates, due to energy loss by improper mirroring of the poles, is recorded using an infrared thermometer.

\section{INVESTIGATION}

The prototype of the maglev system with four electrodynamic rotors was made to study the performance characteristics of the system under varying parameters. The investigation was carried out for 2-pole as well as 8-pole arrangement of the high-strength neodymium magnets. Since the magnets had a cylindrical geometry, it was difficult to achieve a halbach array configuration, hence, updown configuration was employed.

Firstly, the study started with documenting effects of resistivity of the guide plate on the lift, by using four different non-magnetic, conductive materials. These included two aluminium alloys (Al 7075 and Al 6061), commercial aluminium and commercial copper. The air suspension achieved by increasing the rotational speed of the electrodynamic rotors, from 10,000 to $17,500 \mathrm{rpm}$ in successions of $500 \mathrm{rpm}$, for all the materials. It can be observed that since copper has least resistivity among all the four materials, lift obtained at every speed suggests copper to be the most beneficial option of gaining maximum suspension, however, further analysis on the effect of temperature doubts its feasibility, which will be discussed later. To observe the accurate effects, each guide plate were in the same geometrical dimensions, i.e. $1500 \mathrm{~mm} \mathrm{x}$ $1500 \mathrm{~mm}$ while the thickness was $4 \mathrm{~mm}$.

Study was further continued by examining the effect of thickness of the guide plate on the lift of the maglev system, this was achieved by using aluminiumguideplate of five different thicknesses, i.e. $4 \mathrm{~mm}$ to $12 \mathrm{~mm}$ with $2 \mathrm{~mm}$ increments. It is observed that with each increment the air gap between the rotors and the plate increased considerably, however, least differences were observed between plates of $10 \mathrm{~mm}$ and $12 \mathrm{~mm}$ thickness. Similar to the previous experiment, the speed of the rotors was increased from 0 to $17500 \mathrm{rpm}$ in $500 \mathrm{rpm}$ increments.

In a 2-pole arrangement, four magnets are used to create a single pole, while in 8-pole arrangement each magnet is placed in an up-down configuration. It is observed that due to high magnetic flux density of 8-pole arrangement, lift achieved is considerably greater than 2-pole, however, since 8 pole employed up-down configuration, it can be conclusively stated that a 8-pole arrangement in halbach array may result in higher levitation because of its higher magnetic flux density.

Since the resistivity of any given material is directly proportional to the temperature difference, i.e, the resistivity of the material increases with the increase in temperature, silicon being an exception. Since during the working of the maglev system, eddy currents are being generated that create counter magnetic field by mirror the poles of the electrodynamic rotor, this mirroring is imperfect and results in elimination of energy through heat. It can be hypothesized that for huge temperature differences, the lift force may vary resulting in unstable levitation effect. Moreover, the lift height will decrease aggressively which has been observed from the investigation. The experiment was done on $4 \mathrm{~mm}$ thick aluminium and copper plates for a duration of 30 minutes each, where the rotational speed of the rotors were kept constant at 13,500 rpm. An infrared thermometer was used to record temperature differences at 2 minute intervals, and a high capacity LiPo battery was used to run the system for a longer duration.

\section{RESULTS AND DISCUSSION}

A maglev transportation system with four electrodynamic rotros, was designed to study the effects of varying parameters of the rotors on the lift performance of the system. Two rotors were fabricated, one with 2-pole arrangement and the other with 8-pole. The final prototype of the maglev system is presented in figure 5 .

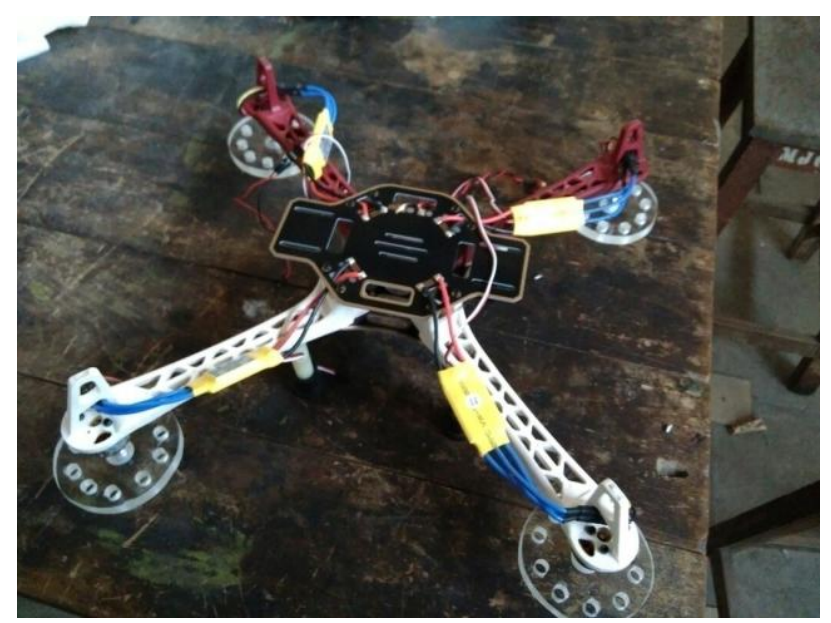

Fig. 5 : Prototype of the maglev system

It has been observed that due to lower resistivity of copper compared to aluminium and its alloys, the system experienced higher levitation displacement reaching up to a height of 5.28 $\mathrm{mm}$ with 17,500 rpm. This have been shown in figure 6 . Further investigation revealed that by increasing the thickness of the guide plate the lift height also increased, this can be due to decrease in resistivity as it is inversely proportional to the thickness. However, the displacement was almost identical for thickness of $10 \mathrm{~mm}$ and $12 \mathrm{~mm}$, hence, it was concluded that the levitation will increase up to a certain limit, after which the levitation height will be inconsequential.

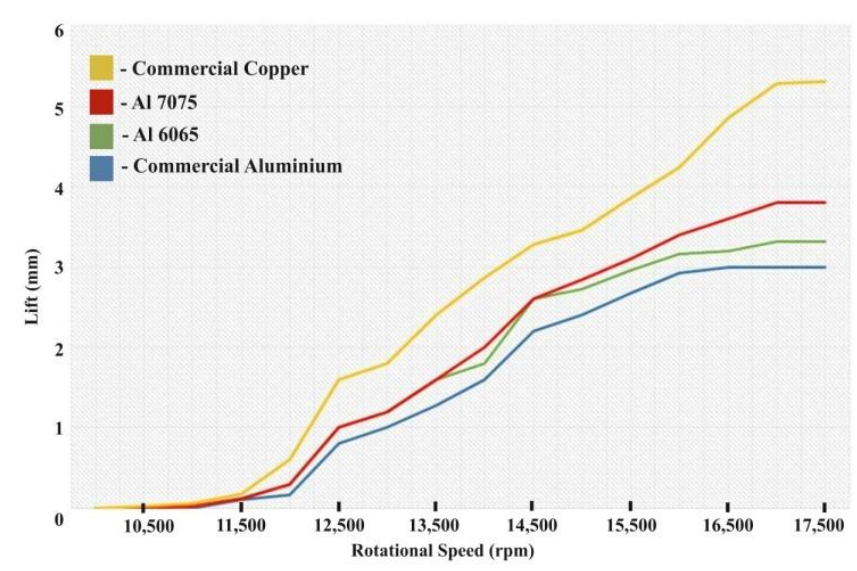

Fig. 6 : Levitation comparison of different materials 


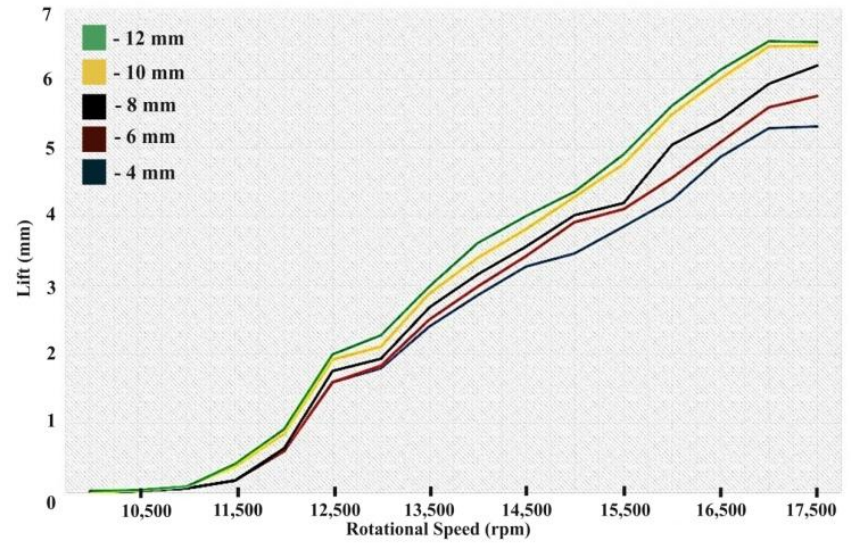

Fig 7 : Levitation comparison of varying thickness of the guide plate

The maximum lift achieved with $12 \mathrm{~mm}$ thickness was $6.52 \mathrm{~mm}$ while for $10 \mathrm{~mm}$ thickness was $6.50 \mathrm{~mm}$, a mere $0.02 \mathrm{~mm}$ difference. $8 \mathrm{~mm}$ thickness yielded $6.16 \mathrm{~mm}$ of lift while $6 \mathrm{~mm}$ thickness gave $5.74 \mathrm{~mm}$ of suspension.

Furthermore, the 8-pole arrangement recorded higher levitation forces while the 2-pole had higher lateral forces. The material selected for was $4 \mathrm{~mm}$ thick commercial copper. 8-pole arrangement yielded $12 \%$ better results, i.e., $5.92 \mathrm{~mm}$ of lift compared to $5.28 \mathrm{~mm}$ of 2-pole arrangement.

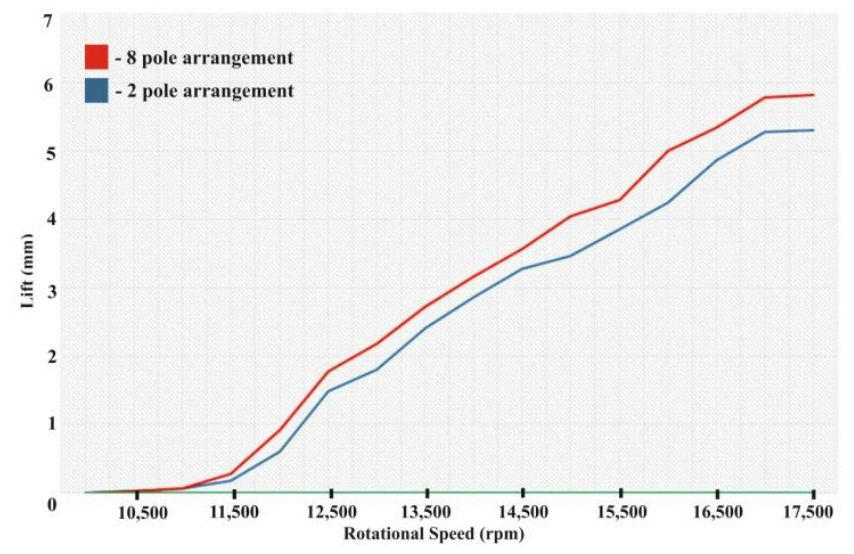

Fig. 8 : Levitation comparison of different magnet orientation

An additional investigation on the effect of temperature change on the levitation height was carried out. Due to improper mirroring of the poles, heat is discharged which is absorbed by the guide plate, copper having a higher thermal conductivity and higher specific heat (in terms of size), it was observed that there was a radical decrease in lift height when the maglev system was kept running for 30 minutes straight at 13,500 rpm. This happened because the resistivity of the metal is directly proportional to the temperature, hence with increase in temperature there was a decrease in lift causing an unstable levitation. Since copper has higher density than aluminium, heat will remain inside it without properly dissipating, which can be eliminated by installing water cooling systems. This is highly infeasible, as copper tracks are already expensive and installing cooling system will further increase the financial strain, hence, thick aluminium track are more practical than copper ones.

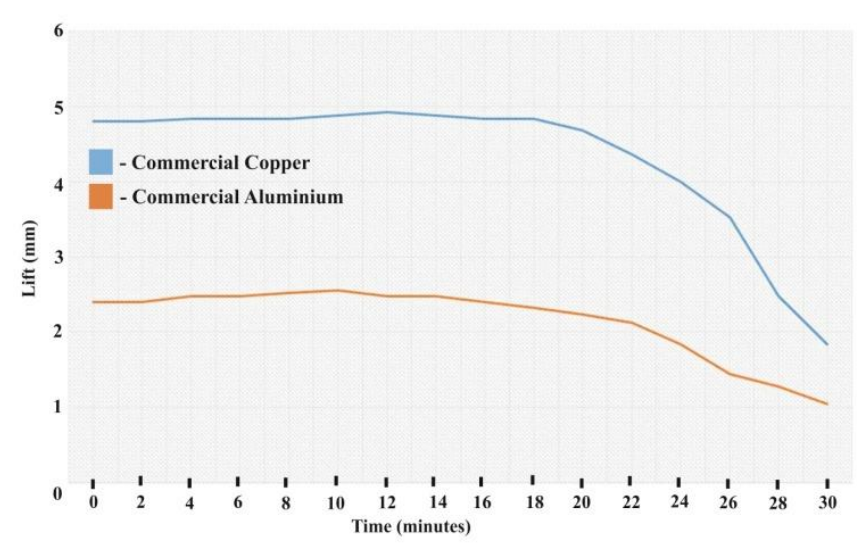

(a)

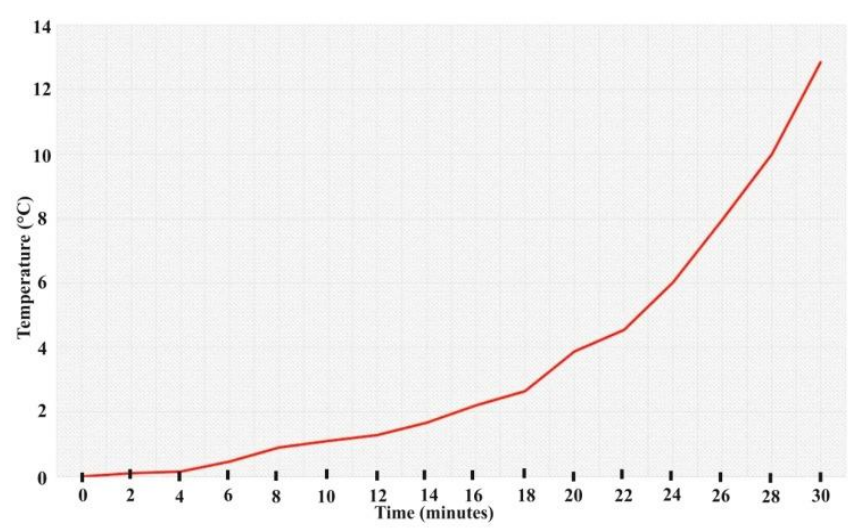

(b)

Fig. 9 : (a) Varying Levitation height over a period of time, (b) Increase in temperature of the guide plate for the same period of time

The maglev quadcopter was levitating for a time period of 30 minutes which resulted in $12.86^{\circ} \mathrm{C}$ of temperature difference when compared to room temperature. The decrease in lift for copper was $61.2 \%$ while that for aluminium was $52.08 \%$. Over 30 mins, the lift difference between copper and aluminium was negligible.

\section{CONCLUSION}

The significance of the paper is that, a maglev planar transportation system was designed and tested through various experimental investigations. As a result, the performance of the maglev system is best achieved by using 8-pole magnetic arrangement with thick aluminium guide plates, and for heavy long term use an air cooling system is recommended. 


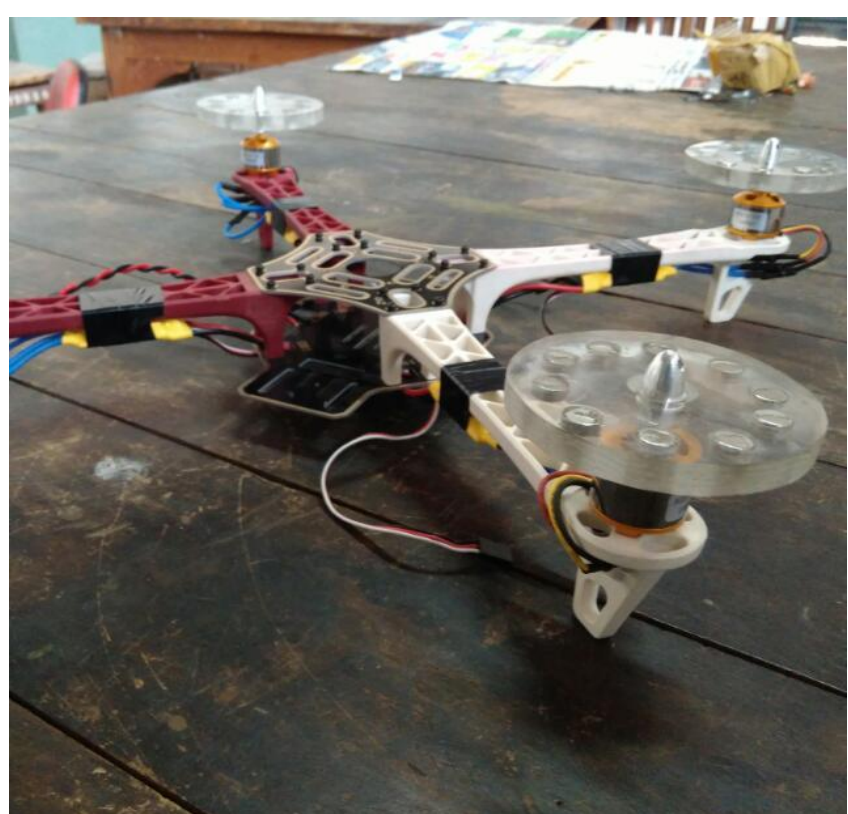

Fig. 10 : Prototype picture of the maglev system along with the $3 \mathrm{D}$ printed electrodynamic rotor

\section{REFERENCES}

[1] Park, Joon-Hyuk\&Baek, Yoon. (2008). Design and Analysis of a Maglev Planar Transportation Vehicle. Magnetics, IEEE Transactions on. 44. 1830 - 1836. 10.1109/TMAG.2008.921953.

[2] J.Bird and T.A.Lipo,"An electrodynamic wheel : An integrated propulsion and levitation machine," Proc. IEEE Int. Electric Machines and Drives Conf., vol. 3, no. 1-4, pp. 1410-1416, June 2003.

[3] N.Fujii,G.Hayashi,andY.Sakamoto,"Characteristics of magnetic lift, propulsion and guidance by using magnet wheels with rotating permanent magnets," in Proc. IEEE Industry Applications Conf. 2000, vol. 1, Oct. 8-12, 2000, pp. 257-262

[4] N. Fujii, K. Ogawa and T. Matsumoto, "Revolving Magnet Wheels with Permanent Magnets," Electrical Eng. in Japan, vol. 1 16, No. 1, pp. 106- 1 18, Jan. 1996 .

[5] N. Fujii, K. Naotsuka, K.Ogawa and T. Matsumoto, "Basic characteristics of magnet wheels with rotating permanent magnets," IEEE IAS 20th Annual Meeting, pp.203-209, Oct. 1994.

[6] N. Fujii, M. Chida, K. Ogawa, "Three Dimensional Force of Magnet Wheel with Revolving Permanent Magnets", IEEE Trans. on Magnetics, vol. 33, no. 5, pp. 4221-4223, 1997. 